\title{
CORRESPONDENCE
}

\section{DAMAGED LIVES}

\section{To the Editor of the Transactions of the Faculty of Actuaries,}

Sir,-Referring to Mr. Low's remarks on the number of damaged lives in the "ultimate" column of a Select Mortality Table (p. 275 of this volume) I have to explain that the figures given in my paper (p. 204) are obtained according to the principle laid down by Dr. T. B. Sprague (J.I. A. xxii. 422), namely that the $l_{x}$ mixed lives aged $x$ in the ultimate column of the $\mathbf{H}^{\mathrm{M}}$ select table consist of $l_{[x]}$ lives who are still select and $l_{x}-l_{[x]}$ damaged lives who will all die in 5 years. Stated in more general terms the principle is that in a table where selection wears off in $n$ years the $l_{x}$ mixed lives in the ultimate column consist of $l_{[x]}$ lives who are still select and $l_{x}-l_{[x]}$ damaged lives who will all die in $n$ years. As Dr. T. B. Sprague's proof of this proposition seems to present some difficulties, a further investigation of the problem may be of interest, and $I$ therefore venture to submit the following considerations in the hope that they may help to make the matter clearer.

Considering the $l_{[x]}$ select lives aged $x$, the table tells us that at the end of $n$ years $l_{x+n}$ of these will survive. It also tells us that out of $l_{x}$ mixed lives $l_{x+n}$ will survive at the end of $n$ years. The table may therefore be considered to exhibit two groups, each containing the same number of lives, namely $l_{x+n}$, who will experience identical rates of mortality in every subsequent year down to the extreme limit of human life. It follows that these two groups must be similarly constituted; that is, each must contain the same number of select lives and the same number of damaged lives. For if the proportion of damaged lives were greater in one group than in the other, the rate of mortality in that group would be higher than in the other.

Now let $\sigma_{x+n}$ and $\delta_{x+n}$ be respectively the number of select lives and of damaged lives in each of the groups; and let $\sigma_{x}$ and $\delta_{x}$ denote respectively the number of select lives and of damaged lives among the $l_{x}$ mixed lives, so that $\sigma_{x}+\delta_{x}=l_{x}$ and $\sigma_{x+n}+\delta_{x+n}=l_{x+n}$. Then out of the $\sigma_{x}$ there will remain after $n$ years $\sigma_{x+n}$ who will be still select, and a number (as yet unknown) who will be damaged. Some of the damaged lives may also survive (the number being as yet unknown) and the sum of these two unknown numbers will be $\delta_{x+n}$. Hence the probability of a select life aged $x$ being still select at the end of $n$ years is $\frac{\sigma_{x+n}}{\sigma_{x}}$. But from a consideration of the $l_{[x]}$ select lives we see that this probability is also $\frac{\sigma_{x+n}}{l_{[x]}}$ and therefore $\frac{\sigma_{x+n}}{l_{[x]}}=\frac{\sigma_{x+n}}{\sigma_{x}}$. It follows that either $\sigma_{x+n}=0$, or $l_{[x]}=\sigma_{x}$.

The first of these equations would imply that after $n$ years of assurance every life must become damaged; but the number of duplicate policies in the " experience" proves that this is impossible. There remains, therefore, only the latter solution, namely, that $l_{[x]}$ is the number of select lives in the "ultimate" column of the table, all the others being therefore damaged.

It may be objected that this reasoning involves the tacit assumption that none of the $\delta_{x}$ damaged lives will become select, and survive as such until the end of the $n$ years. This assumption seems to me not unreasonable, having regard to the fact that damaged lives are necessarily worse than 
select lives, and throughout their existence must be expected to exhibit heavier rates of mortality. As, however, there is room for difference of opinion here, the point may be investigated further. Assume, therefore, that some of the $\sigma_{x+n}$ lives were included in the $\delta_{x}$ damaged lives in existence $n$ years earlier. By considering the $l_{[x]}$ select lives and their survirors after $n$ years we find, as before, that the probability of a select life surviving as "select" $=\frac{\sigma_{x+n}}{l_{[x]}}$. Similarly the probability of a select life surviving as damaged $=\frac{\delta_{x+n}}{l_{[x]}}$. Hence out of the $\sigma_{x}$ select lives there will survive after $n$ years $\sigma_{x} \times \frac{\sigma_{x+n}}{l_{[x]}}$ select, and $\sigma_{x} \times \frac{\delta_{x+n}}{l_{x x]}}$ damaged. But we know from the ultimate column of the table that out of $\sigma_{x}+\delta_{x}$ lives there will survive after $n$ years $\sigma_{x+n}$ select and $\delta_{x+n}$ damaged. Therefore, by subtraction, we see that out of $\delta_{x}$ damaged lives there will survive after $n$ years $\sigma_{x+n}\left(1-\frac{\sigma_{x}}{l_{[x]}}\right)$ select and $\delta_{x+n}\left(1-\frac{\sigma_{x}}{l_{[x]}}\right)$ damaged ; that is to say, the survivors of the damaged lives will consist of select lives and damaged lives mixed in the same proportions as are the survivors of a body of select lives, and therefore they will thereafter experience identically the same rates of deterioration and mortality as the survivors of the select lives. This result seems to me quite inconsistent with the fact that the damaged lives are undoubtedly inferior to the select; for if any of the damaged do become select during the $n$ years we should expect them to be a much smaller proportion of the survirors than if we were dealing with select lives. Looking at this point more closely, we see that the only difference in the subsequent histories of the $l_{[x]}$ select lives and the $l_{x}$ mixed lives is during the first $n$ years, when the mixed lives experience an excess of $l_{x}-l_{[x]}$ deaths. If we leave these $l_{x}-l_{[x]}$ lives out of account, the remainder of the $l_{x}$ mixed lives are found to die, or to survive as select lives, or to survive as damaged lives, in identically the same manner as an equal body of select lives. Now if a body of lives is found to possess identically the ame rates of survirance, of deterioration, and of death, as select lives, that body must necessarily be composed entirely of select lives. Hence the $l_{x}$ nuxed lives are composed of two groups, namely, one numbering $l_{x}-l_{[x]}$ who all die in $n$ years and must be regarded as damaged lives, and the other numbering $l_{[x]}$, who are all select.

In applying this principle it should be remembered that our mortality tables are only approximate, and that selection can in fact be traced (though to a very small extent) for a period longer than is shown by those tables. Further, if the figures in those tables be regarded as approximations of the first order of accuracy, the differences between them will only be approximations of the second order; and as the numbers of damaged lives are found by these differences we cannot place so much reliance upon them as upon the figures relating to select lives.

$$
\text { I am, etc., }
$$

22 George Strent,

A. E. SPRAGUE.

\begin{abstract}
Edingorgh, 6th March 1907.
\end{abstract}

\title{
To the Editor of the Transactions of the Faculty of Actuaries,
}

Srr,-Dr. Ernest Sprague bas been kind enough to show me his letter on this subject. I am much obliged to him for replying so fully to the observations I made, and as the subject is not devoid of interest I should 